\title{
Ten Vestibular Tools for Primary Care
}

\author{
Otto R. Maarsingh* and Vincent A. van Vugt \\ Department of General Practice, Amsterdam University Medical Center (UMC), Vrije Universiteit Amsterdam, Amsterdam \\ Public Health, Amsterdam, Netherlands
}

Keywords: vestibular symptoms, vertigo, dizziness, primary care, general practice, diagnosis, treatment, prognosis

\section{INTRODUCTION}

Although primary care physicians (PCPs) regularly encounter patients with dizziness or vestibular symptoms, they often consider these patients as difficult, challenging or even heartsink $(1,2)$. Given the current scientific evidence and available "vestibular tools," this is unnecessary. We will provide ten vestibular tools that should not be missed, following definition, diagnosis, treatment, and prognosis, respectively (Figure 1).

\section{DEFINITION}

When approaching a potentially complex problem, the use of a uniform nomenclature is crucial. To date, most primary care guidelines use the typology of Drachman and Hart (3). This typology distinguishes four dizziness subtypes, i.e., vertigo (rotational dizziness), presyncope (lightheadedness), disequilibrium (unsteadiness when walking), and non-specific dizziness. The Drachman-Hart typology is primarily based on how patients describe the nature of their symptoms, assuming that this will provide etiological insight, and therefore, diagnostic guidance $(4,5)$. However, both doctors and patients use the term "vertigo" differently $(6-8)$, patients are inconsistent when describing their symptoms (7), the identified subtype does not reliably match the suggested etiology $(5,9)$, and regularly patients have more than one dizziness subtype (10). Therefore, it is time to leave the Drachman-Hart typology and to adopt a more accurate and uniform way to describe vestibular symptoms. The Bárány society, the leading international organization for clinicians and researchers involved in vestibular medicine, previously realized such a nomenclature: the International Classification of Vestibular Disorders (ICVD) $(11,12)$. The ICVD identifies four main vestibular symptoms, i.e., dizziness ("the sensation of disturbed or impaired spatial orientation without a false or distorted sense of motion"); vertigo ("the sensation of self-motion when no motion is present or the sensation of distorted self-motion during normal head movement"); vestibulovisual symptoms ("visual symptoms that result from vestibular pathology or visual-vestibular interaction"); postural symptoms ("balance symptoms related to maintenance of postural stability, occurring only while upright—seated, standing, or walking") (13). These vestibular symptoms are not specific in terms of etiology, not overlapping, and not hierarchical (a single patient can experience multiple symptoms) (13). When assessing a patient with vestibular symptoms, the Bárány society recommends to focus on timing (onset, duration, and evolution of symptom) and triggers (actions, movements, or situations that provoke onset of symptoms) $(11,12)$. Combining the mentioned vestibular symptoms with timing and triggers results in three vestibular syndromes, i.e., acute vestibular syndrome (AVS), episodic vestibular syndrome (EVS), and chronic vestibular syndrome (CVS). AVS is defined as acuteonset, continuous vertigo/dizziness, lasting days to weeks, generally including symptoms that suggest new dysfunction of the vestibular system (like vomiting, nystagmus, and severe postural instability). Disorders presenting with AVS include vestibular neuritis, labyrinthitis, stroke affecting vestibular structures, and traumatic vestibulopathy. EVS is defined as transient vertigo/dizziness 


\section{Definition}

1. Adopt the uniform ICVD nomenclature for the work-up and communication of vestibular symptoms.

\section{Diagnosis}

2. Perform a medication review to identify correctable causes of dizziness.

3. Ask for migraine symptoms and, if present, consider vestibular migraine.

4. Always inquire if there is another contributory cause of dizziness.

5. Use the HINTS bedside test to differentiate between vestibular neuritis and stroke.

6. Avoid standard blood analysis in patients with vestibular symptoms.

\section{Treatment}

7. Use the effective and safe Epley manoeuvre to treat BPPV of the posterior canal.

8. Use effective and safe vestibular rehabilitation to treat chronic vertigo.

9. When treating chronic vertigo, use exercise not drugs.

\section{Prognosis}

10. Employ the 10-item DHI-S questionnaire, not only to assess current handicap and treatment effect, but also to identify patients at risk of an unfavorable outcome.

FIGURE 1 | Ten vestibular tools for primary care.

lasting seconds to hours, generally including symptoms that suggest temporary dysfunction of the vestibular system (like nausea, nystagmus, and sudden falls). Disorders presenting with EVS include vestibular migraine, benign paroxysmal positional vertigo, Menière's disease, and panic attacks. CVS is defined as chronic vertigo/dizziness lasting months to years, generally including symptoms that suggest persistent dysfunction of the vestibular system (like oscillopsia, nystagmus, and gait unsteadiness). Disorders presenting with CVS include poorly compensated vestibulopathy, bilateral vestibulopathy, and persistent postural perceptual dizziness (13). In short, the ICVD nomenclature provides an essential tool for the work-up and communication of vestibular symptoms in primary care (tool \#1).

\section{DIAGNOSIS}

Up to $40 \%$ of patients presenting with vestibular symptoms in primary care remain undiagnosed $(14,15)$. Although this is not unusual for comparable reasons for encounter (like tiredness), we firmly believe it is possible and necessary to reduce the number of undiagnosed dizzy patients in primary care. An accurate diagnosis starts with thorough history taking, focusing on symptom characteristics, timing, and triggers according to the ICVD.

During history taking, the importance of a medication review is apparent. Although an adverse drug effect is a rare cause of vertigo/dizziness in younger patients, it is much more prevalent and regularly missed in older patients. Previous research studies showed that Dutch PCPs scarcely reported adverse drug effect as a cause of dizziness in older patients $(1-3 \%)(14,15)$, whereas a diagnostic panel study among the same population found a much higher proportion (25\%) (10). Drug-induced vertigo can be caused by aminoglycosides, azithromycin, pregabalin, mefloquine, and $\alpha$-blockers, whereas drug-induced dizziness can be caused by anticonvulsants, antidepressants, anti-psychotics, $\beta$ blockers, Calcium channel blockers, antiarrhythmics, diuretics, vasodilators, anxiolytics, and antispasmodics (16-18). As a medication review costs little time and may provide much insight 
(i.e., clues for intervention), it should not be missed in the diagnostic phase (tool \#2). During such a review, a practical guide may help to rapidly identify potential adverse drug effects (19).

A more common cause of episodic vertigo is vestibular migraine (VM). This is a migraine variant with vestibular symptoms and poorly understood pathophysiology. Despite its prevalence and high impact on healthcare cost and utilization, VM remains clinically underdiagnosed (20). The diagnostic criteria for VM include the presence or history of migraine, at least five episodes with vestibular symptoms of moderate or severe intensity and at least $50 \%$ of episodes associated with migraine features (i.e., headache, motion sensitivity, photo- or phonophobia, or visual aura) (21). We recommend physicians to structurally ask for migraine symptoms (tool \#3), and, if present, consider vestibular migraine (VM).

Another small but effective tool, especially regarding older patients, is to incorporate the following question in your diagnostic work-up: Is there another contributory cause of dizziness? (tool \#4) According to a diagnostic panel study among 417 older dizzy patients in Dutch primary care, $62 \%$ had two or more contributory causes of dizziness (10). Among a consecutive cohort of 621 patients in tertiary care (average age 56 years, range $11-90$ years), $30 \%$ of dizzy patients had more than one diagnosis (22).

If the history reveals red flags (e.g., neurological symptoms, new headache, or acute deafness) (23), it is important to minimize the probability of a central cause of dizziness. However, when sharpening one's diagnostic tools, population awareness is crucial: the prior probability of a central cause of dizziness in a primary care population is very low compared to secondary/tertiary care. In a study cohort that consisted of patients hospitalized with isolated vertigo, the risk for stroke during 4-year follow-up was 3.01-times higher compared to the general population; vertigo patients with three or more risk factors (including age >55 years, male gender, hypertension, diabetes, coronary artery disease, and hyperlipidemia) even had a 5.51-fold higher for stroke $(24,25)$. However, in a surveillance study among patient presenting with dizziness symptoms to the emergency department, only $0.7 \%$ with isolated dizziness symptoms had a stroke/TIA (26). According to the ecology of medical care (27), this proportion will be even lower for patients presenting with the same symptoms in primary care. In case of an acute vestibular syndrome (i.e., rapid onset of vertigo, nausea/vomiting, and gait unsteadiness in association with headmotion intolerance and nystagmus) another promising tool comes in: the three-step Head Impulse-Nystagmus-Test of Skew (HINTS) exam (HINTS; tool \#5). The HINTS exam is a simple bedside test that is relatively easy to learn (https:// medicinetoday.com.au/vertigovideos). The HINTS exam can help differentiate between vestibular neuritis and stroke, because the presence of any of three oculomotor signs (normal horizontal head impulse; gaze-direction nystagmus; or skew deviation) indicates a central cause of acute vestibular syndrome. A recent systematic review revealed that the HINTS exam has a pooled sensitivity of $96 \%$ and specificity of $71 \%$ to detect stroke (28), which indicates an even higher diagnostic accuracy than early MRI (29).
When revising one's diagnostic tools, it is important to reconsider overrated tools. According to an observational study $(n=2,812)$, Dutch PCPs performed blood analyses in $22 \%$ of older patients presenting with dizziness (15). Until present, there is no scientific evidence that standardized blood analysis has additional value during the work-up of vestibular symptoms. Among 4,538 patients included in etiologic studies, laboratory abnormalities that explained dizziness were limited to three patients with electrolyte disturbances, 11 with glucose disorders, 11 with anemia, and one with hypothyroidism (30). In a community based study, the results of standardized blood analysis among 149 dizzy subjects and 97 controls did not differ (31). Therefore, use blood tests only on a strict medical indication and avoid standard blood analysis in patients with vestibular symptoms (tool \#6).

\section{TREATMENT}

A very rewarding vestibular tool is the Epley maneuver (tool \#7). This is a relatively simple, safe, and highly effective treatment for the most prevalent cause of episodic vertigo, i.e., benign paroxysmal positional vertigo of the posterior canal-a diagnosis that can be confirmed by using the Dix-Hallpike test (https:// www.youtube.com/watch?v=kEM9p4EX1jk\&feature=youtu.

be) (32). Despite its proven effectiveness, PCPs have not yet embraced the Epley maneuver. During a survey among 426 PCPs, only 57\% used the Epley maneuver. The most common reason (50\%) for PCPs not to use the maneuver was that they did not know how to perform the technique (33). The second reason (30\%) was not being convinced of its effectiveness. Both deserve reconsideration, as the Epley maneuver can be easily learned (https://medicinetoday.com.au/vertigovideos) and the scientific evidence is convincing [Epley vs. sham maneuver, complete resolution of vertigo: OR 4.42 (95\% CI 2.62-7.44); Epley vs. sham maneuver, conversion of Dix-Hallpike: OR 9.62 (95\% CI 6.0-15.42)] (32).

Another effective, safe, and neglected tool is vestibular rehabilitation (VR; tool \#8). VR is an exercise based treatment that gradually stimulates the vestibular system and vestibular compensation (34). Chronic vertigo occurs when natural vestibular compensation fails (35). Although a clear definition is lacking (36), chronic vertigo is-based on clinical course and expert opinion-often defined as symptoms persisting more than 1 month $(17,37)$. In primary care, physicians can refer patients to a specialized physiotherapist for VR. Despite the scientific evidence for VR, $<10 \%$ of PCPs in the Netherlands and UK reported its use $(33,38)$. As this may be due to a lack of availability or access to VR (38), the University of Southampton developed a freely available online VR intervention (https:// balance.lifeguidehealth.org). This online VR intervention was investigated among two different cohorts in primary care $(n$ $=296$ and $n=322$, respectively), showing both reduction of dizziness and dizziness-related impairment after 3 and 6 months $(39,40)$. Being easily accessible, safe, effective and low cost, online VR has the potential to substantially improve the quality of life for a largely undertreated group of patients. 
Although nowadays VR is the preferred treatment for chronic vertigo according to US, Dutch, and UK clinical practice guidelines (17, 41-43), anti-vertigo drugs like betahistine are still regularly prescribed. According to a large observational study, betahistine was initially prescribed to more than two thirds of vertigo patients in general practice and was still being used after 6 months (44). This enthusiastic prescribing contradicts with the state of the science, though, as a recent Cochrane review showed only weak evidence for the effectiveness of betahistine to treat chronic vertigo (45). Also, long term prophylactic treatment with betahistine does not change the time course of vertigo episodes related to Meniere's disease compared with placebo (46). When using GRADE methodology to compare VR (4 RCTs, $n=565$ adults with different causes of chronic vertigo) with betahistine (11 RCTs, $n=606$ adults with different causes of chronic vertigo), there is a difference in effectiveness and quality of evidence: vertigo patients receiving VR reported higher improvement compared to sham/no treatment [odds ratio 2.67 (95\% CI 1.853.86 ); moderate quality of evidence], whereas vertigo patients treated with betahistine reported limited improvement compared to placebo [risk ratio 1.30 (95\% CI 1.05-1.60); low quality of evidence]. In contrast to $\mathrm{VR}$, none of the betahistine trials was conducted in primary care, which limits the generalizability (47). In short, when treating chronic vertigo, use exercise not drugs (tool \#9).

\section{PROGNOSIS}

Until present, many risk factors of handicapping dizziness and/or vertigo have been identified, like chronic dizziness, daily dizziness, activity limitation or avoidance due to dizziness, anxiety or depression, polypharmacy, and impaired functional mobility (48-51). One of the most powerful predictors of an unfavorable course of dizziness, though, is significant impairment at baseline as measured with the Dizziness Handicap

\section{REFERENCES}

1. Luxon LM. Evaluation and management of the dizzy patient. $J \quad$ Neurol Neurosurg Psychiatry. (2004) 75(Suppl 4):iv4552. doi: 10.1136/jnnp.2004.055285

2. Salmon P, Peters S, Clifford R, Iredale W, Gask L, Rogers A, et al. Why do general practitioners decline training to improve management of medically unexplained symptoms? J Gen Intern Med. (2007) 22:56571. doi: 10.1007/s11606-006-0094-z

3. Drachman DA, Hart CW. An approach to the dizzy patient. Neurology. (1972) 22:323-34. doi: 10.1212/WNL.22.4.323

4. Edlow JA. Diagnosing dizziness: we are teaching the wrong paradigm! Acad Emerg Med. (2013) 20:1064-6. doi: 10.1111/acem.12234

5. Newman-Toker DE, Dy FJ, Stanton VA, Zee DS, Calkins H, Robinson KA. How often is dizziness from primary cardiovascular disease true vertigo? A systematic review. J Gen Intern Med. (2008) 23:208794. doi: 10.1007/s11606-008-0801-z

6. Blakley BW, Goebel J. The meaning of the word "vertigo." Otolaryngol Head Neck Surg. (2001) 125:147-50. doi: 10.1067/mhn.2001.117869

7. Newman-Toker DE, Cannon LM, Stofferahn ME, Rothman RE, Hsieh YH, Zee DS. Imprecision in patient reports of dizziness symptom quality: a cross-sectional study conducted in an acute
Inventory (DHI) (49, 52). The DHI is a 25 -item selfreport questionnaire, developed to measure impairment due to vestibular symptoms (53). Nowadays, it has been translated in at least 17 languages and considered to be the most used vestibular PROM (54). However, the length of the DHI limits its use in daily clinical practice. Therefore, the abbreviated 10-item DHI$S$ questionnaire (fill-in time $\pm 2 \mathrm{~min}$ ) was developed in 1998 (55). During a psychometric evaluation in primary care, the DHI$S$ showed excellent criterion validity, test-retest reliability, and responsiveness (56). Recently, a prediction study with external validation in primary care showed that the ability of the DHI-S to identify patients at risk of an unfavorable course of dizziness improved when combined with the predictors age, history of arrhythmia, and looking up as a provoking factor (area under the curve after external validation $=0.78)(52)$. Given the fact that -in addition to its prognostic qualities - the DHI-S provides information on current handicap and can be used to monitor treatment effect, we believe that this questionnaire should not be missed in the vestibular toolkit of the PCP (tool \#10).

\section{CONCLUSIONS}

In this article, we present ten vestibular tools for primary care. PCPs can use these tools to improve diagnosis, treatment, and prognosis of vestibular symptoms. All tools are readily available and do not require intensive training. By simplifying proper management of vestibular symptoms, we hope that PCPs will embrace dizziness as an exciting symptom.

\section{AUTHOR CONTRIBUTIONS}

$\mathrm{OM}$ and VV wrote and approved the manuscript. All authors contributed to the article and approved the submitted version. care setting. Mayo Clin Proc. (2007) 82:1329-40. doi: 10.4065/82.11. 1329

8. Stanton VA, Hsieh YH, Camargo CA Jr, Edlow JA, Lovett PB, et al. Overreliance on symptom quality in diagnosing dizziness: results of a multicenter survey of emergency physicians. Mayo Clin Proc. (2007) 82:131928. doi: 10.4065/82.11.1319

9. Lawson J, Johnson I, Bamiou DE, Newton JL. Benign paroxysmal positional vertigo: clinical characteristics of dizzy patients referred to a falls and syncope unit. QJM. (2005) 98:357-64. doi: 10.1093/qjmed/hci057

10. Maarsingh OR, Dros J, Schellevis FG, van Weert HC, van der Windt DA, ter $\mathrm{RG}$, et al. Causes of persistent dizziness in elderly patients in primary care. Ann Fam Med. (2010) 8:196-205. doi: 10.1370/afm.1116

11. Bisdorff A, Von Brevern M, Lempert T, Newman-Toker DE. Classification of vestibular symptoms: towards an international classification of vestibular disorders. J Vestib Res. (2009) 19:1-13. doi: 10.3233/VES-2009-0343

12. Bisdorff AR, Staab JP, Newman-Toker DE. Overview of the international classification of vestibular disorders. Neurol Clin. (2015) 33:541-50. doi: 10.1016/j.ncl.2015.04.010

13. Bisdorff A. Vestibular symptoms and history taking. Handbook Clin Neurol. (2016) 137:83-90. doi: 10.1016/B978-0-444-63437-5.00006-6

14. Maarsingh OR, Dros J, Schellevis FG, van Weert HC, Bindels PJ, van der Horst HE. Dizziness reported by elderly patients in family practice: 
prevalence, incidence, and clinical characteristics. BMC Fam Pract. (2010) 11:2. doi: 10.1186/1471-2296-11-2

15. Stam $H$, Harting $T$, Sluijs $M$, Marum R, Horst $H$, Wouden JC, et al. Usual care and management of fall risk increasing drugs in older dizzy patients in Dutch general practice. Scand J Prim Health Care. (2016) 34:16571. doi: 10.3109/02813432.2016.1160634

16. Geneesmiddelgeïnduceerde draaiduizeligheid [Drug-induced vertigo]. Gebu. (2015) 49:51-6. https://www.ge-bu.nl/en/article/drug-induced-vertigo?full

17. Bouma M, De Jong J, Dros J, Maarsingh OR, Moormann KA, Smelt AFH, et al. Dutch Guideline on Dizziness [NHG-Standaard Duizeligheid]. Huisarts Wet. (2017) 60:348-56. https://www.henw.org/system/files/download/HW60-348. pdf

18. Shoair OA, Nyandege AN, Slattum PW. Medication-related dizziness in the older adult. Otolaryngol Clin North Am. (2011) 44:455-71. doi: 10.1016/j.otc.2011.01.014

19. Altissimi G, Colizza A, Cianfrone G, de Vincentiis M, Greco A, Taurone $\mathrm{S}$, et al. Drugs inducing hearing loss, tinnitus, dizziness and vertigo: an updated guide. Eur Rev Med Pharmacol Sci. (2020) 24:7946-52. doi: 10.26355/eurrev_202008_22477

20. Huang TC, Wang SJ, Kheradmand A. Vestibular migraine: An update on current understanding and future directions. Cephalalgia. (2020) 40:10721. doi: $10.1177 / 0333102419869317$

21. Lempert T, Olesen J, Furman J, Waterston J, Seemungal B, Carey J, et al. Vestibular migraine: diagnostic criteria. J Vestib Res. (2012) 22:16772. doi: 10.3233/VES-2012-0453

22. van Leeuwen RB, Colijn C, van Wensen E, Bruintjes TD. The dizzy patient: consider a second diagnosis. Neurologist. (2017) 22:69-71. doi: 10.1097/NRL.0000000000000116

23. Barraclough K, Bronstein A. Vertigo. BMJ. (2009) 339:b3493. doi: 10.1136/bmj.b3493

24. Lee CC, Su YC, Ho HC, Hung SK, Lee MS, Chou P, et al. Risk of stroke in patients hospitalized for isolated vertigo: a four-year followup study. Stroke. (2011) 42:48-52. doi: 10.1161/STROKEAHA.110. 597070

25. Choi KD, Lee H, Kim JS. Vertigo in brainstem and cerebellar strokes. Curr Opin Neurol. (2013) 26:90-5. doi: 10.1097/WCO.0b013e32835c5edd

26. Kerber KA, Brown DL, Lisabeth LD, Smith MA, Morgenstern LB. Stroke among patients with dizziness, vertigo, and imbalance in the emergency department: a population-based study. Stroke. (2006) 37:24847. doi: 10.1161/01.STR.0000240329.48263.0d

27. Green LA, Fryer GE Jr, Yawn BP, Lanier D, Dovey SM. The ecology of medical care revisited. $N$ Engl J Med. (2001) 344:2021-5. doi: 10.1056/NEJM200106283442611

28. Krishnan K, Bassilious K, Eriksen E, Bath PM, Sprigg N, Brækken SK, et al. Posterior circulation stroke diagnosis using HINTS in patients presenting with acute vestibular syndrome: a systematic review. Eur Stroke J. (2019) 4:233-9. doi: 10.1177/2396987319843701

29. Kattah JC, Talkad AV, Wang DZ, Hsieh YH, Newman-Toker DE. HINTS to diagnose stroke in the acute vestibular syndrome: three-step bedside oculomotor examination more sensitive than early MRI diffusion-weighted imaging. Stroke. (2009) 40:3504-10. doi: 10.1161/STROKEAHA.109. 551234

30. Hoffman RM, Einstadter D, Kroenke K. Evaluating dizziness. Am J Med. (1999) 107:468-78. doi: 10.1016/S0002-9343(99)00260-0

31. Colledge NR, Barr-Hamilton RM, Lewis SJ, Sellar RJ, Wilson JA. Evaluation of investigations to diagnose the cause of dizziness in elderly people: a community based controlled study. BMJ. (1996) 313:788-92. doi: 10.1136/bmj.313.7060.788

32. Hilton MP, Pinder DK. The Epley (canalith repositioning) manoeuvre for benign paroxysmal positional vertigo. Cochrane Database Syst Rev. (2014) 2014:Cd003162. doi: 10.1002/14651858.CD003162.pub3

33. van Vugt VA, Diaz Nerio PM, van der Wouden JC, van der Horst HE, Maarsingh OR. Use of canalith repositioning manoeuvres and vestibular rehabilitation: a GP survey. Scand J Prim Health Care. (2017) 35:1926. doi: 10.1080/02813432.2017.1288683

34. McDonnell MN, Hillier SL. Vestibular rehabilitation for unilateral peripheral vestibular dysfunction. Cochrane Database Syst Rev. (2015) 1:CD005397. doi: 10.1002/14651858.CD005397.pub4
35. Lacour M, Helmchen C, Vidal PP. Vestibular compensation: the neuro-otologist's best friend. J Neurol. (2016) 263(Suppl. 1):S54-64. doi: 10.1007/s00415-015-7903-4

36. Sloane PD, Coeytaux RR, Beck RS, Dallara J. Dizziness: state of the science. Ann Intern Med. (2001) 134:82332. doi: 10.7326/0003-4819-134-9_Part_2-200105011-00005

37. Brandt T, Huppert T, Hüfner K, Zingler VC, Dieterich M, Strupp M. Longterm course and relapses of vestibular and balance disorders. Restor Neurol Neurosci. (2010) 28:69-82. doi: 10.3233/RNN-2010-0504

38. Jayarajan V, Rajenderkumar D. A survey of dizziness management in general practice. J Laryngol Otol. (2003) 117:599604. doi: 10.1258/002221503768199915

39. Geraghty AWA, Essery R, Kirby S, Stuart B, Turner D, Little P, et al. Internetbased vestibular rehabilitation for older adults with chronic dizziness: a randomized controlled trial in primary care. Ann Fam Med. (2017) 15:20916. doi: $10.1370 / \mathrm{afm} .2070$

40. van Vugt VA, van der Wouden JC, Essery R, Yardley L, Twisk JWR, van der Horst HE, et al. Internet based vestibular rehabilitation with and without physiotherapy support for adults aged 50 and older with a chronic vestibular syndrome in general practice: three armed randomised controlled trial. BMJ. (2019) 367:15922. doi: 10.1136/bmj.15922

41. Bhattacharyya N, Gubbels SP, Schwartz SR, Edlow JA, El-Kashlan H, Fife T, et al. Clinical practice guideline: benign paroxysmal positional vertigo (update). Otolaryngol Head Neck Surg. (2017) 156:S1-47. doi: 10.1177/019459981668 9667

42. National Institute for Health and Care Excellence. Clinical Knowledge Summaries: Vestibular neuronitis. (2011). https://cks.nice.org.uk/vestibularneuronitis.

43. Hall CD, Herdman SJ, Whitney SL, Cass SP, Clendaniel RA, Fife TD, et al. Vestibular rehabilitation for peripheral vestibular hypofunction: an evidence-based clinical practice guideline: from the American physical therapy association neurology section. $J$ Neurol Phys Ther. (2016) 40:124-55. doi: 10.1097/NPT.000000000000 0120

44. Agus S, Benecke H, Thum C, Strupp M. Clinical and demographic features of vertigo: findings from the revert registry. Front Neurol. (2013) 4:48. doi: 10.3389/fneur.2013.00048

45. Murdin L, Hussain K, Schilder AG. Betahistine for symptoms of vertigo. Cochrane Database Syst Rev. (2016) 2016:Cd010696. doi: 10.1002/14651858.CD010696. pub2

46. Adrion C, Fischer CS, Wagner J, Gürkov R, Mansmann U, Strupp M. Efficacy and safety of betahistine treatment in patients with meniere's disease: primary results of a long term, multicentre, double blind, randomised, placebo controlled, dose defining trial (BEMED trial). BMJ. (2016) 352:h6816. doi: 10.1136/bmj.h6816

47. van Vugt VA, van der Horst HE, Payne RA, Maarsingh OR. Chronic vertigo: treat with exercise, not drugs. BMJ. (2017) 358:j3727. doi: 10.1136/bmj.j 3727

48. Bailey KE, Sloane PD, Mitchell M, Preisser J. Which primary care patients with dizziness will develop persistent impairment? Arch Fam Med. (1993) 2:847-52. doi: 10.1001/archfami.2.8.847

49. Dros J, Maarsingh OR, van der Windt DA, Oort FJ, ter RG, de Rooij SE, et al. Functional prognosis of dizziness in older primary care patients: a prospective cohort study. J Am Geriatr Soc. (2012) 60:2263-9. doi: 10.1111/jgs.12031

50. Kroenke K, Lucas C, Rosenberg ML, Scherokman B, Herbers JE. One-year outcome for patients with a chief complaint of dizziness. J Gen Intern Med. (1994) 9:684-9. doi: 10.1007/BF02599010

51. Nazareth I, Yardley L, Owen N, Luxon L. Outcome of symptoms of dizziness in a general practice community sample. Fam Pract. (1999) 16:6168. doi: $10.1093 /$ fampra/16.6.616

52. Stam H, Maarsingh OR, Heymans MW, van Weert H, van der Wouden JC, van der Horst HE. Predicting an unfavorable course of dizziness in older patients. Ann Fam Med. (2018) 16:428-35. doi: 10.1370/afm.2289

53. Jacobson GP, Newman CW. The development of the dizziness handicap inventory. Arch Otolaryngol Head Neck Surg. (1990) 116:424-7. doi: 10.1001/archotol.1990.0187004004 6011 
54. Van De Wyngaerde KM, Lee MK, Jacobson GP, Pasupathy K, RomeroBrufau S, McCaslin DL. The component structure of the dizziness handicap inventory (DHI): a reappraisal. Otol Neurotol. (2019) 40:121723. doi: 10.1097/MAO.0000000000002365

55. Jacobson GP, Calder JH. A screening version of the dizziness handicap inventory (DHI-S). Am J Otol. (1998) 19:804-8.

56. van Vugt VA, de Vet HCW, van der Wouden JC, van Weert H, van der Horst HE, Maarsingh OR. The 25-item dizziness handicap inventory was shortened for use in general practice by 60 percent. J Clin Epidemiol. (2020) 126:56-64. doi: 10.1016/j.jclinepi.2020.06.021
Conflict of Interest: The authors declare that the research was conducted in the absence of any commercial or financial relationships that could be construed as a potential conflict of interest.

Copyright $\odot 2021$ Maarsingh and van Vugt. This is an open-access article distributed under the terms of the Creative Commons Attribution License (CC BY). The use, distribution or reproduction in other forums is permitted, provided the original author(s) and the copyright owner(s) are credited and that the original publication in this journal is cited, in accordance with accepted academic practice. No use, distribution or reproduction is permitted which does not comply with these terms. 